\title{
Charge Ordering and Phase Separations in the Spinless Fermion Model with Repulsive Intersite Interaction
}

\author{
W.R. Czart, S. Robaszkiewicz and B. Tobijaszewska \\ Institute of Physics, A. Mickiewicz University \\ Umultowska 85, 61-614 Poznań, Poland
}

\begin{abstract}
We study a simple effective model for description of charge orderings in narrow band materials, i.e. the spinless fermion model with repulsive intersite interaction $W$. The analysis is concentrated on the problem of phase separations and the effects of next-nearest neighbor hopping $t_{2}$ on the charge ordered states in this system. The cases of $d$-dimensional $(d \geq 2)$ hypercubic lattices are considered for arbitrary particle concentration $(0<n<1)$. Within the broken symmetry Hartree-Fock approximation the phase diagrams as a function of $W$ and $n$ are evaluated for representative cases. The results for $t_{2} \neq 0$ are compared with those found for the case with nearest neighbor hopping only.
\end{abstract}

PACS numbers: 71.10.Fd, 71.30.+h, 71.45.Lr, 64.75.Gh

\section{Introduction}

Electron charge orderings $(\mathrm{CO})$ phenomena are currently under intense investigations as the subject is relevant to a broad range of important materials, including among others manganites, cuprates and numerous organic conductors [1-6]. Here we will discuss a simple effective model for description of $\mathrm{CO}$ in narrow band systems, i.e. the spinless fermion model with repulsive intersite interaction $W$.

The model Hamiltonian has the following form:

$$
\begin{aligned}
& \widehat{H}=\sum_{i j} t_{i j} c_{i}^{+} c_{j}+\frac{1}{2} \sum_{i j} W_{i j} n_{i} n_{j}-\mu \sum_{i} n_{i}, \\
& n=\frac{1}{N} \sum_{i}\left\langle n_{i}\right\rangle, \quad 0<n<1,
\end{aligned}
$$

where $c_{i}^{+}\left(c_{i}\right)$ are the creation (annihilation) operators for spinless (or completely spin polarized) fermions on site $i, n_{i}=c_{i}^{+} c_{i}, t_{i j}$ are the single particle hopping 
integrals (between the nearest neighbors $t$ and the next nearest neighbors $t_{2}$ ), $\mu$ - the chemical potential, $W_{i j}$ are the intersite density-density interactions, assumed to be repulsive $\left(W_{i j}>0\right)$ and restricted to nearest neighbors.

The physical systems which can be reliably described with the model include strongly polarized materials, such as ${ }^{3} \mathrm{He}$ in strong magnetic field and electron systems where one spin-band is filled (and only electrons of one spin species are itinerant). Moreover, the model can also serve as an approximation (for charge degrees of freedom) of the extended Hubbard model in the limit of infinite on-site repulsion (the equivalence is exact in one dimension only).

Apart from a few papers [5-8] the research so far concentrates on the case of half-filled band with nearest-neighbor hopping.

We have performed extensive study of the phase diagrams and thermodynamic properties of this model for arbitrary $n$ and various lattice structures $(2 \leq d \leq \infty)$ [9]. In the analysis we have used the (broken symmetry) HartreeFock approximation (HFA), which for the models with intersite interactions only, can yield rigorous results for $d=\infty$, since in such a case only the Hartree diagrams survive in the perturbation theory $[7,10]$.

We have focused on the problem of phase separations (PS) and the effects of next-nearest-neighbor hopping $\left(t_{2}\right)$ on the charge ordered states in these systems. Below we only shortly summarize the main findings and present selected phase diagrams evaluated for a few representative cases at $T=0$ and $T>0$.

\section{Results and discussion}

Denotations used in the following: In the case of $d=2$ SQ lattice and $d=3$ SC lattice: $W_{0}=2 d W / t, b=t_{2} / t$, whereas in the case of $d=\infty$ hypercubic lattice: $W_{0}=W^{*} / t^{*}, b=t_{2}^{*} / t^{*}$, where $W^{*}$ and $t^{*}, t_{2}^{*}$ are defined by $W=W^{*} / 2 d$, $t=t^{*} / \sqrt{2 d}, t_{2}=t_{2}^{*} / \sqrt{2 d(d-1)}$, to ensure that the hopping and the intersite interaction remains finite in the limit of infinite dimensions [10].

\subsection{Results for $t_{2}=0$}

For $n=0.5$, in the case of $d \geq 2$ hypercubic lattices the ground state (GS) is found to be charge ordered $(\mathrm{CO})$ for any $W_{0}>0$, which is in agreement with the renormalization group results for $d=2$ and $d=3$ lattices [11].

Beyond $n=0.5$, in all these cases the GS diagrams consist of two states: the non-ordered state (NO) if $W_{0}<W_{\mathrm{c} 0}(n)$ and the phase separated (PS) state: $\mathrm{CO} / \mathrm{NO}$, if $W_{0}>W_{\mathrm{c} 0}(\mathrm{n})$. (see e.g. Figs. 1a, b, plotted for SQ lattice and $d=\infty$ lattice, respectively). The transition at $W_{\mathrm{c} 0}(\mathrm{n})$ is discontinuous (of the 1 st order) for any $n$, and the value of $W_{\mathrm{c} 0}(\mathrm{n})$ strongly increases with the increase in the deviation from $n=0.5$.

With increasing $T$ the systems can exhibit several different types of behavior (cf. Fig. 2 and Ref. [9]): (i) a sequence of two transitions $\mathrm{PS}(\mathrm{CO} / \mathrm{NO}) \rightarrow \mathrm{CO} \rightarrow$ $\mathrm{NO}$, (ii) a single 1st order transition $\mathrm{PS} \rightarrow \mathrm{NO}$, (iii) a single 2nd order transition $\mathrm{CO} \rightarrow \mathrm{NO}$ (at $n=0.5$ only). In definite ranges of $n$ and $W_{0}$ one finds also 

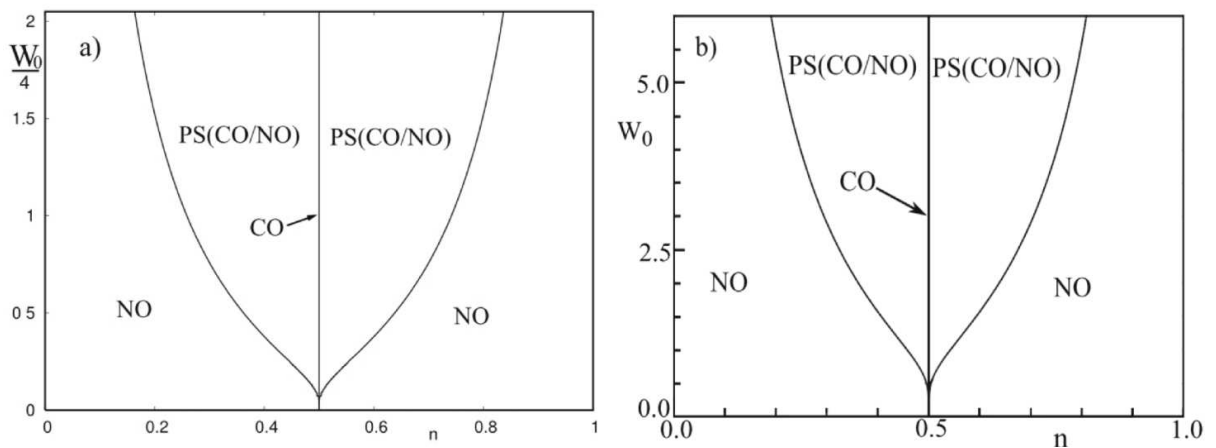

Fig. 1. Ground state phase diagrams as a function of $n$ for $t_{2}=0$. The phase separated region is marked $\mathrm{PS}(\mathrm{CO} / \mathrm{NO}$ ) (a) for $d=2 \mathrm{SQ}$ lattice; (b) for $d=\infty$. Solid curves: the first order boundary between $\mathrm{PS}(\mathrm{CO} / \mathrm{NO})$ and nonordered state $(\mathrm{NO})$. The pure $\mathrm{CO}$ is stable at $n=0.5$ only.

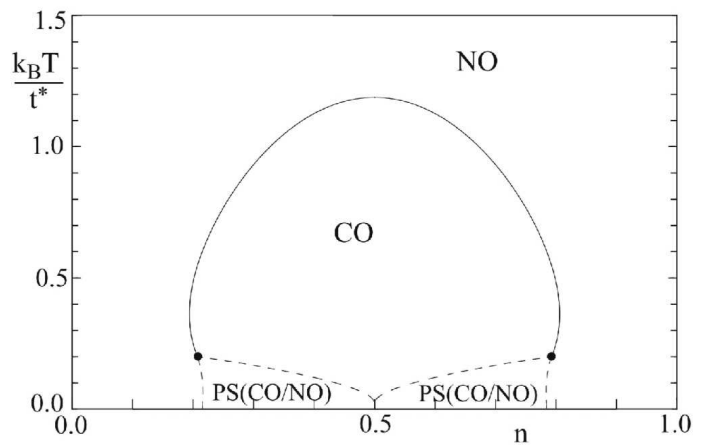

Fig. 2. Finite temperature phase diagram plotted for $d=\infty, t_{2}=0$ and $W_{0}=5.0$. Solid and dashed curves denote the second order and first order transition lines, respectively. Filled dots denote the tricritical points (TCP).

the reentrant charge-order phenomena (see Fig. 2), i.e. (iv) a sequence of three transitions: $\mathrm{NO} \rightarrow \mathrm{PS} \rightarrow \mathrm{CO} \rightarrow \mathrm{NO}$ and (v) a sequence of two second-order transitions: $\mathrm{NO} \rightarrow \mathrm{CO} \rightarrow \mathrm{NO}$.

\subsection{The effects of frustration $\left(t_{2} \neq 0\right)$}

At half-filling the frustration introduced by $t_{2}$ suppresses the perfect nesting instability towards $\mathrm{CO}$ at small $W_{0}$.

In Fig. 3 we show the ground state diagrams evaluated for $t_{2} \neq 0$ ( $W_{0}$ vs. $b=t_{2} / t$ ) where we use the following denotations: COM - metallic CO state (the band gap is zero, but the CO parameter $\left.n_{Q}=(1 / N) \sum_{i \sigma}\left\langle n_{i, \sigma}\right\rangle \mathrm{e}^{\mathrm{i} Q R_{i}} \neq 0\right)$, COI insulating $\mathrm{CO}$ state and $\mathrm{NOM}$ - nonordered metallic state. As we see, the form of these diagrams strongly depends on the lattice dimensionality:

(i) For $d=2$ and $d=3$ lattices (cf. Fig. 3a, c) the critical interactions $W_{\mathrm{c} 0}$ necessary to stabilize the CO state decrease rather slowly with decreasing $t_{2}$ for 


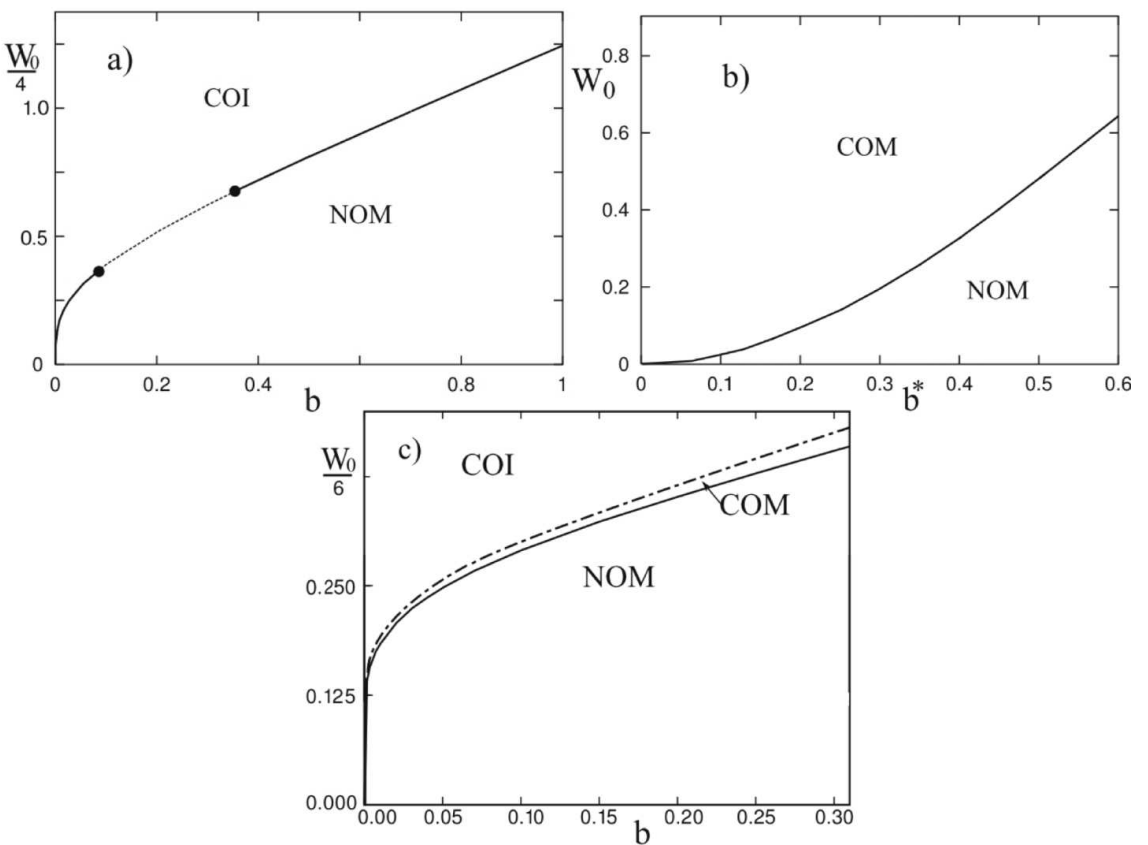

Fig. 3. Ground state phase diagrams ( $W_{0}$ vs. $b$ ) for $n=0.5$. Solid and dashed curves denote, respectively, the second order and first order transition lines between the $\mathrm{CO}$ and NO states. (a) $d=2 \mathrm{SQ}$ lattice. Filled dots denote points where the first-order transition line and the second-order transition lines meet. The discontinuous NOM-COI transition occurs for $0.085<b<0.355$. Narrow regions of metallic CO phase (COM) are not plotted in this diagram. (b) $d=\infty$ hypercubic lattice. (c) $d=3$ SC lattice. Dashed-dotted line: values of $W_{0}$ where the band gap closes. Outside the COM region the system is either in a charge order insulator phase (COI) or in a nonordered metal (NOM) state.

small $b\left[\left(W_{\mathrm{c} 0}\right)^{-1} \sim|\ln (b)|\right]$, whereas for $d=\infty$ lattice (Fig. 3b) the decreasing is much faster $\left(W_{\mathrm{c} 0} \sim b^{2}\right)$.

(ii) For $d=3$ and $d=\infty$ lattices the transition at $W_{\mathrm{c} 0}$ is of the 2 nd order for any $b=t_{2} / t$ (Fig. 3b, c). On the contrary for $d=2$ lattice the continuous transition occurs only for $b<0.085$ and $b>0.355$, whereas for $0.085<b<0.355$ the transition at $W_{\mathrm{c} 0}$ is discontinuous.

(iii) For $d=2$ and $d=3$ lattices and $t_{2} \neq 0$ the continuous transition at $W_{\mathrm{c} 0}$ is to the metallic COM state and only further increase in $W_{0}$ yields the transition to the insulating COI state. Thus, one has a sequence of transitions with increasing $W_{0}: \mathrm{NOM} \rightarrow \mathrm{COM} \rightarrow \mathrm{COI}$ (cf. Fig. 3c). On the contrary, in the case of discontinuous transitions to the $\mathrm{CO}$ state $(d=2$ lattice, $0.085<b<0.355)$ the ordered state is insulating for any $W_{0}>W_{\mathrm{c} 0}$ and one observes a single first-order transition with increasing $W_{0}: \mathrm{NOM} \rightarrow$ COI at $W_{\mathrm{c} 0}$. 
(iv) For $d=\infty$ lattice there is never a true band gap and one has a single second-order transition with increasing $W_{0}: \mathrm{NOM} \rightarrow \mathrm{COM}$ at $W_{\mathrm{c} 0}$ (Fig. $3 \mathrm{~b}$ ).

With increasing $T$ at $n=0.5$ and $t_{2} \neq 0$ one can observe several different types of behavior, depending on $W_{0}$ and $b$ :

(i) a single second-order transition: $\mathrm{COM} \rightarrow$ NOM. It occurs if the ground state is COM. For $d=\infty$ this is the case for any $W_{0}>W_{\mathrm{c} 0}$, whereas for $d=2$ and $d=3$ lattices such a situation takes place in rather narrow ranges of $W_{0}$ (cf. Fig. 3c).

(ii) a sequence of two transitions: $\mathrm{COI} \rightarrow \mathrm{COM} \rightarrow \mathrm{NOM}$.

(iii) a single first-order transition: $\mathrm{COI} \rightarrow \mathrm{NOM}$. It can occur for $d=2$ lattice, if $0.085<b<0.355$, for $W_{0}$ close to $W_{\mathrm{c} 0}(b)\left(W_{0}>W_{\mathrm{c} 0}(b)\right)$.

(iv) the system remains in the NOM state at any $T$. It occurs for any $W_{0}<W_{\mathrm{c} 0}(\mathrm{~b})$ for all $d \geq 2$ lattices.

As concerns the effects of frustration beyond $n=0.5$, we have found that the structure of the GS diagrams can be substantially changed in comparison to that obtained for $t_{2}=0$ (Fig. 1). In particular one finds [9] that:

(i) for $t_{2} \neq 0$ in definite ranges of $n$ and $W_{0}$ there can exist at $T=0$ not only the PS and NO states, but also a homogeneous CO phase.

(ii) For the considered values of $t_{2} / t$ the phase boundaries of this phase with the NO state are of the second order, whereas those with the PS states are of first order.

We have confined our study to the two-sublattice CO case and do not consider a possible incommensurate ordering. Important next steps in the investigations would be to take into account the states involving incommensurate charge orderings (CDW with $\boldsymbol{q} \neq \boldsymbol{Q}$ ) as well as various types of striped phases and "island type" phases, which can eventually appear in the presence of longer-range interactions, and to extend analysis to the "more exotic" lattice structures, like the diamond one, anisotropic triangular ones, etc.

\section{References}

[1] E. Dagotto, T. Hotta, A. Moreo, Phys. Rep. 344, 1 (2001).

[2] T. Goto, B. Lüthi, Adv. Phys. 52, 67 (2003).

[3] R. Micnas, J. Ranninger, S. Robaszkiewicz, Rev. Mod. Phys. 62, 113 (1990).

[4] T. Takahashi, Y. Nogami, K. Yakushi, J. Phys. Soc. Jpn. 75, 51008 (2006).

[5] M.Yu. Kagan, K.I. Kugel, Physics-Uspekhi 44, 553 (2001).

[6] P. Fulde, P. Thalmeier, G. Zwicknagel, Solid State Phys. 60, 1 (2006) and references therein.

[7] G.S. Uhrig, R. Vlaming, Phys. Rev. Lett. 71, 271 (1993).

[8] Y.Z. Zhang, M.T. Tran, V. Yushankhai, P. Thalmeier, Eur. Phys. J. B 44, 265 (2005).

[9] W.R. Czart, S. Robaszkiewicz, B. Tobijaszewska, in preparation. 
[10] E. Müller-Hartmann, Z. Phys. B 74, 507 (1989); W. Metzner, D. Vollhardt, Phys. Rev. Lett. 62, 324 (1989).

[11] R. Shankar, Rev. Mod. Phys. B 66, 129 (1994). 